\title{
The role of endoscopic ultrasonography in transmural drainage/debridement of walled-off pancreatic necrosis
}

\author{
Mateusz Jagielski, Marian Smoczyński, Krystian Adrych \\ Department of Gastroenterology and Hepatology, Medical University of Gdansk, Gdansk, Poland
}

Gastroenterology Rev 2018; 13 (2): 160-162

DOI: https://doi.org/10.5114/pg.2018.72608

Address for correspondence: Mateusz Jagielski MD, PhD, Department of Gastroenterology and Hepatology, Medical University of Gdansk, 17 Smoluchowskiego St, 80-214 Gdansk, Poland, phone: +48 5834936 40, fax: +48 58349 36 50, e-mail: matjagiel@gmail.com

In numerous publications it has been proved that application of endoscopic ultrasonography (EUS) in transmural drainage of pancreatic fluid collections (PFCs) increases the efficiency and safety of treatment [1-3]. In our article "The role of endoscopic ultrasonography in endoscopic drainage/debridement of walledoff pancreatic necrosis - a single-center experience" published in 2015 in "Pancreatology" we compared two groups of patients with symptomatic walled-off pancreatic necrosis (WOPN) treated endoscopically with and without the use of EUS in our medical center between 2001 and 2013 [4]. In the mentioned article the group of patients treated with the use of EUS (conventional drainage) until 2011 consisted of 112 people, while the number of patients treated endoscopically with EUS from the time of introduction of EUS in our hospital in 2011 until 2013 was 64. As a result of comparison of the two groups we demonstrated that the use of endoscopic ultrasonography during endoscopic drainage/debridement of WOPN significantly reduces the number of procedure-related complications. However, it has no influence on the duration of treatment or the efficiency of therapy. In the time being (2017) the number of patients who have been treated with EUS-guided drainage/debridement of WOPN in our department since 2011 is 114 . The increased number of patients treated endoscopically with the use of EUS inspired us to share our observations. Endoscopic ultrasonography allows one to identify solid debris in the lumen of the pancreatic collection, which enables one to distinguish WOPN from other pancreatic fluid collections. What is more, EUS-guided drainage/debridement of WOPN not only facilitates the choice of fistula site, but also allows drainage of necrotic collections regardless of their location (particularly in the absence of an endoscopically defined area of extrinsic compression). The application of endoscopic ultrasonography in drainage/debridement of walled-off pancreatic necrosis significantly reduces the number of procedure-related complications, especially gastrointestinal bleeding. Thereby it increases the safety of treatment, which was described in our article. All the above-mentioned advantages of EUS in the treatment of patients with pancreatic necrosis make it extremely useful for transmural drainage/debridement of WOPN. However, EUS also has disadvantages such as technical failures. Under endoscopic view during the conventional drainage we are able to choose the optimal approach to the necrotic collections with use of a therapeutic endoscope or duodenoscope. The entry approach to WOPN with the echoendoscope in the course of EUS-guided drainage is often more difficult. The fistula performed under endosonographic view and with the help of an echoendoscope, in contrast to a standard endoscope (conventional drainage), often has a hard location, which makes the approach to the fistula more difficult or even impossible in further treatment, after the decompression of the necrotic collection. The hard approach to the fistula requires time to find the optimal position of the endoscope in the lumen of the gastrointestinal tract, which often extends the duration of the next endoscopic procedures, after performing gastrocystostomy or duodenocystostomy with EUS guidance. It is often necessary to approach the fistula in the long scope position (a gastric loop is left in the stomach - Figure 1) or even in inversion (Figure 2).

The advantages of endoscopic ultrasonography in transmural drainage of PFCs are unquestionable (Figures 3 and 4), which should make the use of EUS during transmural drainage/debridement of WOPN obligatory, even despite the mentioned technical failures. Never- 


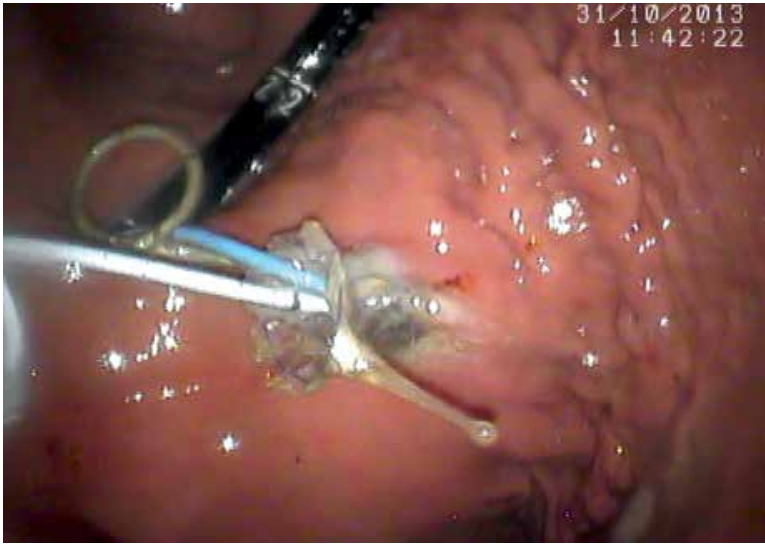

Figure 1. Transgastric endoscopic drainage/ debridement. The transmural self-expandable metallic stent (SEMS), stent 'double pigtail' $7 \mathrm{Fr}$ and nasal drain $7 \mathrm{Fr}$, inserted through the stoma into the area of pancreatic necrosis. Difficult approach to fistula in the long scope position

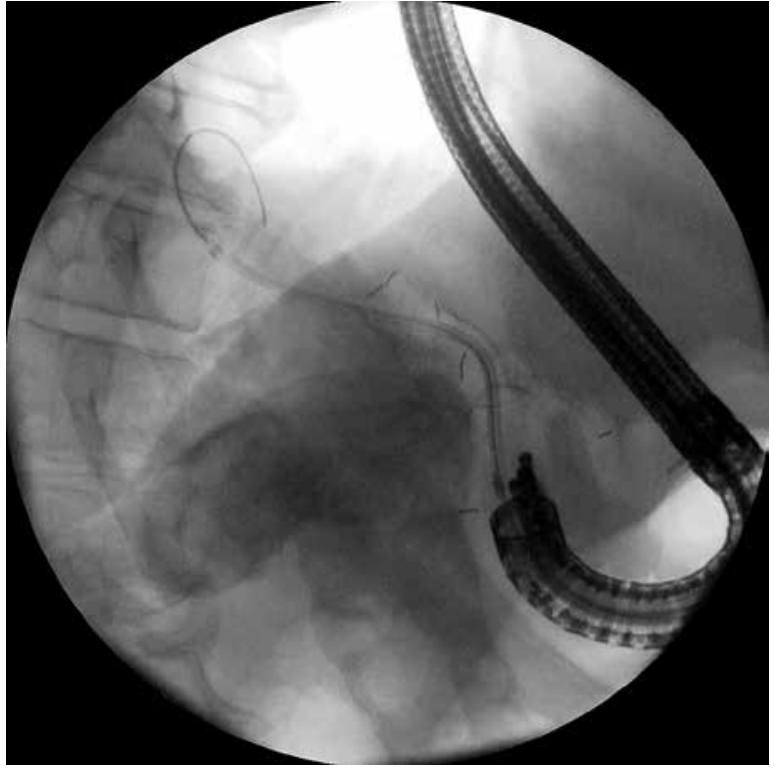

Figure 2. Transgastric endoscopic drainage/debridement. The transmural self-expandable metallic stent (SEMS) is visible in the fluoroscopic image. Difficult approach to fistula in the inversion

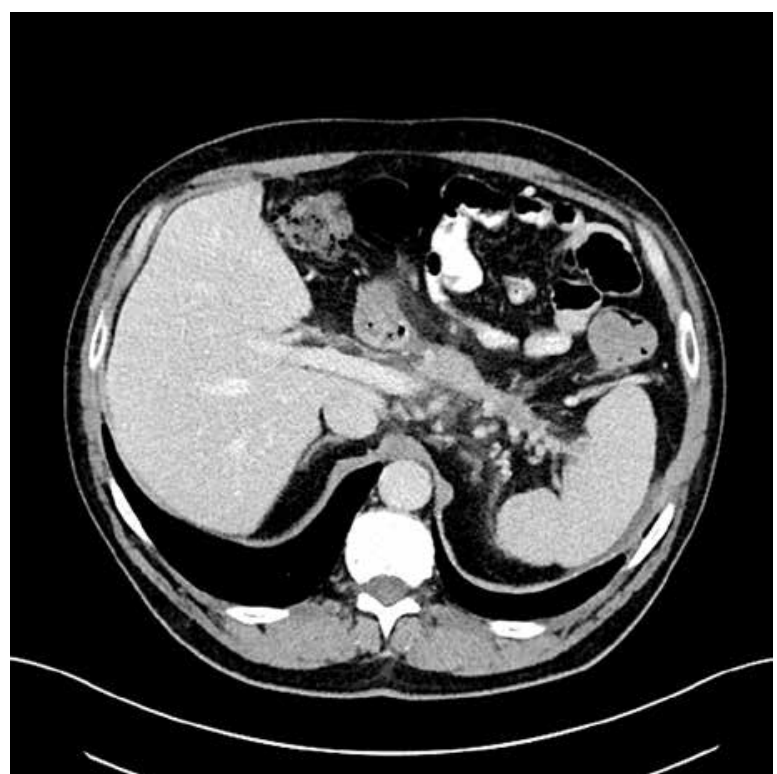

Figure 4. Contrast-enhanced computed tomography performed after the end of endoscopic treatment showed complete regression of WOPN necrosis visible in contrast-enhanced computed tomography (CECT) during endoscopic transmural drainage/debridement ( $8^{\text {th }}$ day of drainage)

theless, we still hold the view that in some patients with symptomatic WOPN the lack of EUS does not exclude performance of transmural drainage/debridement. The technically harder entry approach during
EUS-guided drainage in comparison to conventional drainage means that EUS-guided drainage requires extensive experience from the endoscopist. The learning curve of this procedure is very long. 


\section{Conflict of interest}

The authors declare no conflict of interest.

\section{References}

1. Varadarajulu S, Wilcox CM, Tamhane A, et al. Role of EUS in drainage of peripancreatic fluid collections not amenable for endoscopic transmural drainage. Gastrointest Endosc 2007; 66: 1107-19.

2. Varadarajulu S, Christein JD, Tamhane A, et al. Prospective randomized trial comparing EUS and EGD for transmural drainage of pancreatic pseudocysts (with videos). Gastrointest Endosc 2008; 68: 1102-11.

3. Park DH, Lee SS, Moon SH, et al. Endoscopic ultrasound-guided versus conventional transmural drainage for pancreatic pseudocysts: a prospective randomized trial. Endoscopy 2009; 41: 842-8.

4. Jagielski M, Smoczynski M, Jablonska A, et al. The role of endoscopic ultrasonography in endoscopic debridement of walledoff pancreatic necrosis - a single center experience. Pancreatology 2015; 15: 503-7.

Received: 19.09.2017

Accepted: 11.12 .2017 